\title{
DETERMINISTIC AND STOCHASTIC ANALYSIS OF THE LITHIATION/DELITHIATION DYNAMICS OF A CATHODE NANOPARTICLE
}

CHANG WANG*, CAMERON L. HALL*, AND PETER D. HOWELL*

Abstract. We use asymptotic methods to analyse the effects of discreteness and random noise on a mathematical model of the lithiation/delithiation dynamics of a single electrode nanoparticle. We begin by presenting a simple ordinary differential equation model for the lithiation dynamics of a nanoparticle under voltage control, assuming that the nanoparticle is sufficiently small that internal diffusion and phase-separation can be neglected. We use the method of matched asymptotic expansions to show that the dynamics of this single particle system can be reduced to rapid switching between an 'empty' state and a 'full' state, and we identify the critical voltage at which the switch between states occurs under both quasi-equilibrium and out-of-equilibrium conditions. Next we present an alternative model of nanoparticle lithiation, assuming that a large but finite number of 'lithiation states' are available and that transitions between states are governed by the discrete chemical master equations. This leads to a discrete, stochastic model of lithiation that has the capacity to exhibit very different behavior from the original differential equation model. We use discrete-to-continuum asymptotic methods to analyse the discrete, stochastic model, and we identify the crucial dimensionless parameter that controls whether or not the discreteness and thermal noise play an important role in the dynamic behavior of the system.

1. Introduction. Lithium iron phosphate (LFP) is a cheap, energetically efficient, and environmentally friendly cathode material that is widely used in lithium-ion batteries that operate at moderate voltages [22]. Studies of LFP cathodes (see, for example, $[11,15,19])$ indicate that optimal performance is best achieved by constructing cathodes from a large number of carbon-coated nanoparticles. However, both theoretical studies and experimental work have demonstrated that the dominant reactions and rate-limiting steps in an electrode nanoparticle are very different from those in larger particles. For example, it has been found that phase separation into lithiumrich and lithium-poor regions is suppressed in very small particles [6, 13, 18, 23], and that the lithiation rate is controlled by surface reactions rather than transport within nanoparticles $[1,23]$.

These observations suggest that the concentration of lithium within a sufficiently small nanoparticle can be treated as being spatially uniform. This so-called pseudocapacitor limit significantly simplifies the mathematical modeling of LFP dynamics [12] compared to a large number of recent models (see [3] for a recent review), which use phase-field methods based on a homogenisation of the Cahn-Hilliard equations to describe LFP electrochemistry, and to the early 'shrinking-core' models of lithiation/delithiation based on Stefan models (see, for example, [22]). While the small size of nanoparticles may enable some valuable modeling simplifications, it also introduces some complications. Since nanoparticles may host just a few tens of thousands of lithium atoms, it is possible that the discreteness of the available 'lithiation states' may be important and thus a continuum concentration variable may not be appropriate to represent the amount of intercalated lithium. Moreover, thermal fluctuations in the amount of intercalated lithium in a very small particle may have a significant effect on that particle's lithiation dynamics.

Systems of coupled nanoparticles are known to exhibit an unusual type of phase separation, sometimes referred to as a mosaic instability [20]. Based on both mathematical models and experimental observations, Dreyer et al. $[9,10]$ proposed that

* Mathematical Insitute, University of Oxford, Andrew Wiles Building, Oxford OX2 6GG, UK (hall@maths.ox.ac.uk, howell@maths.ox.ac.uk) 
most particles in a nano-particulate lithium iron phosphate (LFP) cathode tend to be either 'full' or 'empty' of lithium at any particular point in time (especially when charging/discharging happens slowly). Rather than phase separation between lithiated and unlithiated regions within each particle, it appears that there is phase separation on the many-particle scale [5, 9, 10, 20]. As noted in [9], this is analogous to a coupled system of balloons subject to a controlled pressure; such systems tend to have a range of stable and unstable steady states, and random noise has the potential to have a dramatic effect on their dynamics.

The above discussion prompts the questions: (1) What is an appropriate model of the noise experienced by a many-particle electrode? (2) What effects does such noise have on the dynamics of the system? As a first step towards answering these questions, we present a detailed analysis of the dynamics of the lithium concentration within a single electrode nanoparticle subject to voltage-controlled lithiation. We compare and contrast a classical deterministic model (which uses a continuum lithium concentration variable) with a stochastic model (which uses a discrete lithium concentration variable), to determine when and where discrete and/or stochastic phenomena are important, and to explore how stochastic fluctuations in lithium concentration might be included in future many-particle models of electrode dynamics.

We begin in section 2 by developing a simple continuum model of the lithiation dynamics of a single nanoparticle. Following [8, 20], we assume that the lithiumcontaining nanoparticle is sufficiently small that all phase-separation effects are suppressed, and hence the free energy associated with the normalized lithium concentration is given by a classical double well potential. We then assume that the lithium concentration evolves deterministically according to the Butler-Volmer equation. We use asymptotic methods to analyse in detail a model dynamic problem in which the electric potential is increased at a constant rate. Our analysis reveals that the rapid jump from the nanoparticle being 'empty' to being 'full' occurs a short time after what would be predicted from a purely equilibrium analysis. However, the dynamics of this transition is very delicate, and it might reasonably be argued that a small amount of stochastic noise added to the deterministic model might alter the dynamics completely.

Therefore, in section 3, we introduce a discrete, probabilistic model of the lithium concentration in a nanoparticle based on the chemical master equation. In sections 4 and 5, we perform an asymptotic analysis of the discrete, probabilistic model subject a time-dependent applied potential. We find that the effects of both discreteness and stochasticity are controlled by a single dimensionless parameter $\alpha$, and we derive simplified leading-order models valid for limiting values of $\alpha$. When $\alpha \ll 1$, discrete effects become negligible, and the continuous deterministic model from section 2 is recovered. At the other extreme where $\alpha \gg 1$, the system is inherently discrete and cannot be described by the classical continuum theory. In the intermediate case where $\alpha=O(1)$, both discrete effects and continuum effects are important and are described by a hybrid discrete-continuum model.

In summary, our analysis shows how random noise can be self-consistently incorporated into a model of lithiation dynamics, and confirms that discreteness and thermal noise can have a noticeable effect on the nature and timing of the jump from 'empty' to 'full'.

\section{Deterministic model of lithium intercalation.}

2.1. Model formulation. In this section, we develop and analyse a deterministic model of the lithiation of a single electrode nanoparticle based on the Butler- 


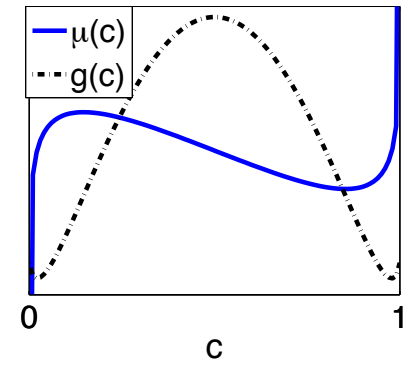

Fig. 1: The double-well free energy, $g(c)$, given by (1), and the associated N-shaped chemical potential, $\mu(c)$, given by (2). In these plots, $k_{B} T / \Omega=0.25$.

Volmer equation. Throughout this paper, we assume that lithium ions are abundant in the surrounding electrolyte, that there are no limitations based on the rate of reaction at the reference electrode or auxiliary electrode, and that there is no competition with reactions at other nanoparticles in the working electrode. Thus, we concentrate on the simplified problem where the applied electric potential at a nanoparticle is specified, and we observe the changing lithium content of the nanoparticle in response to this potential. Possible extensions of the present model, such as coupling together many nanoparticles into a single electrode, will be discussed in section 6 .

Following [20], we consider a lithium-containing nanoparticle that is sufficiently small for all phase-separation effects are suppressed, and hence the free energy associated with the normalized lithium concentration, $c$, is given by the classical double well potential:

$$
g(c)=\Omega c(1-c)+k_{\mathrm{B}} T[c \log (c)+(1-c) \log (1-c)] .
$$

The normalized concentration, $c$, is scaled so that $c=1$ corresponds to a nanoparticle saturated with lithium; $\Omega$ is the scaled enthalpy of mixing, $k_{B}$ is Boltzmann's constant, and $T$ is the temperature. Equation (1) is a classical result that can be found in [8,20], for example, or can be obtained from the models described in $[3,21]$ and related works by eliminating all terms that depend on spatial gradients of $c$.

The associated chemical potential for the free energy (1) is

$$
\mu(c)=\frac{\mathrm{d} g}{\mathrm{~d} c}=\Omega(1-2 c)+k_{\mathrm{B}} T \log \left(\frac{c}{1-c}\right) .
$$

Provided $2 k_{\mathrm{B}} T<\Omega$ (which is the case in all practical problems), this chemical potential is 'N-shaped', as shown in Figure 1, indicating that three equilibrium concentrations of lithium are possible for certain values of the applied potential.

The Butler-Volmer equation [4] relates the current density, $I$, to the overpotential, $\eta$, by

$$
I(\eta)=I_{0}\left[\exp \left(\frac{a e}{k_{\mathrm{B}} T} \eta\right)-\exp \left(-\frac{(1-a) e}{k_{\mathrm{B}} T} \eta\right)\right],
$$

where $a \in(0,1)$ is the charge transfer coefficient, $e$ is the charge on an electron, and $I_{0}$ is the exchange current density. Following convention [4], we assume that charge transfer is symmetric, so that $a=1 / 2$. Moreover, we treat $I_{0}$ as a constant; in some 
cases, $I_{0}$ can be a function of the ion concentration, and the effect of including this in our model is discussed in Appendix A.

The overpotential $\eta$ is defined as the difference between the electrode potential and a constant equilibrium potential. In the half-cell setting of this paper, the overpotential depends on the difference between the applied potential over the nanoparticle interface, $E(t)$, and the chemical potential of the nanoparticle, $\mu(c)$. Specifically, we follow [20] to obtain

$$
\eta(t)=E(t)-\frac{\mu(c(t))}{e} .
$$

The current density, $I$, is in turn related to the flux of lithium in or out of the nanoparticle. Net charge conservation implies that

$$
I= \pm \frac{e \rho V}{S} \frac{\mathrm{d} c}{\mathrm{~d} t},
$$

where $V$ and $S$ are the volume and surface area of the particle, and $\rho$ is the lattice density (the number of potential sites for intercalated lithium per unit volume). The positive sign should be taken in the case of a cathode nanoparticle (where positive current density corresponds to flux of lithium into the nanoparticle), while the negative sign should be taken in the case of an anode nanoparticle.

We note that $g(c)$ is a symmetric function of $c$, in that $g(c) \equiv g(1-c)$, and that setting $a=1 / 2$ means that $I(-\eta) \equiv-I(\eta)$. These symmetries mean that lithiation and delithiation are mathematically equivalent, and similarly that cathode particles and anode particles are mathematically equivalent. Thus, without loss of generality, we will concentrate on the lithiation of a cathode particle. We non-dimensionalize the equations above using the intrinsic time-scale $t_{\text {int }}=e V \rho / S I_{0}$, which corresponds to the time required to deplete a fully-lithiated particle at constant current density $I_{0}$. Additionally, we rescale energy with $\Omega$, and potential with $\Omega / e$ to obtain the dimensionless Butler-Volmer model:

$$
\frac{\mathrm{d} c}{\mathrm{~d} t}=\sinh \left(\frac{E(t)-\mu(c(t))}{2 \epsilon}\right), \quad \mu(c)=1-2 c+\epsilon \log \left(\frac{c}{1-c}\right) .
$$

The parameter

$$
\epsilon=\frac{k_{\mathrm{B}} T}{\Omega}
$$

measures the relative importance of entropy and enthalpy, and it controls the shape of the function $\mu(c)$. Typically we expect $\epsilon$ to be small, so that at a stable equilibrium the nanoparticle is either nearly empty of lithium $(c \approx 0)$ or nearly full of lithium $(c \approx 1)$. Below we will carry out asymptotic analysis in the limit $\epsilon \rightarrow 0$. This analysis reveals the generic behavior to be expected of solutions to the model (6), and guides the subsequent efforts in sections 3 to 5 to incorporate discrete and stochastic effects.

2.2. Equilibria. When $E$ varies sufficiently slowly, the nanoparticle has enough time to exchange lithium ions freely with electrolyte until its concentration satisfies $\mu(c)=E$, so that forward and reverse reactions balance. In such a situation, the system is always in quasi-steady-state, and its concentration simply follows equilibria of the system (6). Depending on the value of $E$, there is either one stable equilibrium (when $E>\mu^{*}$ or $E<\mu^{* *}$ ), or one unstable equilibrium and two stable equilibria 


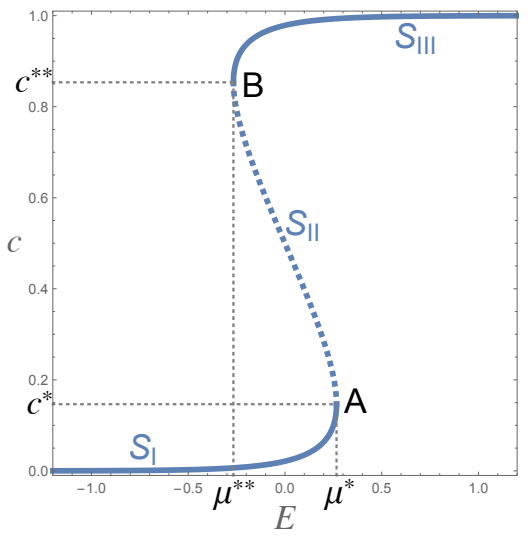

(a)

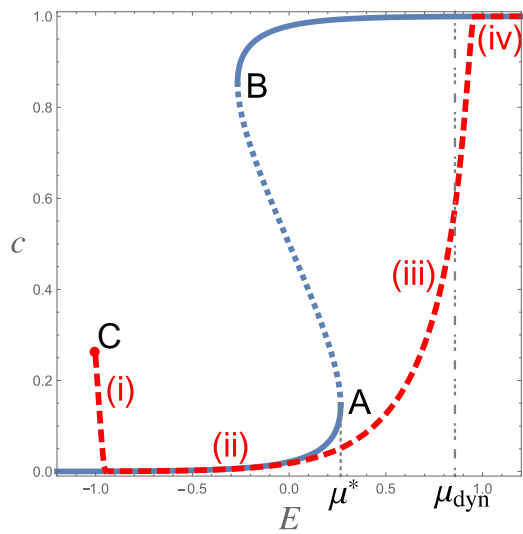

(b)

Fig. 2: (a) Equilibria of the Butler-Volmer system (6), plotted with $\epsilon=0.25$. The three equilibrium branches $\left(S_{I}, S_{I I}\right.$ and $\left.S_{I I I}\right)$ are connected by saddle-node bifurcations at $A$ and B. (b) Numerical solution (red, dot-dashed) of the initial-value problem (9) with $\epsilon=0.25, t_{0}=2$ and $c_{0}=0.25$, shown alongside the equilibrium curve. As described in the text, the dynamic behavior is separated into four stages (i) to (iv).

(when $\mu^{* *}<E<\mu^{*}$ ), as shown in Figure 2(a), where the three equilibrium branches are labeled $S_{I}, S_{I I}$ and $S_{I I I}$.

It is clear that this system exhibits hysteresis as $E$ passes through the critical values $\mu^{*}$ and $\mu^{* *}$. There are saddle-node bifurcations at the two critical points $A$ and $\mathrm{B}$, whose coordinates are approximately given by

$$
\left(c^{*}, \mu^{*}\right) \sim\left(\frac{\epsilon}{2}, 1-\epsilon \log (2 / \epsilon)-\epsilon\right), \quad\left(c^{* *}, \mu^{* *}\right) \sim\left(1-\frac{\epsilon}{2},-1+\epsilon \log (2 / \epsilon)+\epsilon\right)
$$

as $\epsilon \rightarrow 0$, with corrections of order $\epsilon^{2}$.

Based on this equilibrium analysis, we would expect that if $E(t)$ is increased slowly, starting at a value less than $\mu^{* *}$, then $c(t)$ will remain on the lower stable 'empty' branch $\mathrm{S}_{\mathrm{I}}$ and then jump to the higher 'full' branch $\mathrm{S}_{\text {III }}$ as $E$ passes through $\mu^{*}$. Conversely, if $E(t)$ is decreased slowly, starting at a value greater than $\mu^{*}$, then we would expect $c(t)$ to remain on $\mathrm{S}_{\mathrm{III}}$ and then jump to the lower branch $\mathrm{S}_{\mathrm{I}}$ as $E$ passes through $\mu^{* *}$. In realistic problems, however, the applied potential may change at a reasonable rate compared to the intrinsic timescale, $t_{\text {int }}$. Hence, it is important to understand the hysteretic behavior under realistic dynamic conditions.

2.3. Out-of-equilibrium dynamics. When the applied potential $E(t)$ varies on a time scale similar to or smaller than the intrinsic time, $t_{\text {int }}$, we need to solve a dynamic problem to understand the out-of-equilibrium behavior. As noted above, the problem (6) is invariant under the transformation $c \mapsto 1-c$ and $E \mapsto-E$, and so no generality is lost in considering only an increasing $E(t)$. Specifically, we set $E(t)=1+\lambda t$, where the constant $\lambda$ measures how fast the applied potential increases relative to the intrinsic timescale $t_{\text {int }}$, while we expect the transition from the low branch $S_{\text {I }}$ to the high branch $S_{\text {III }}$ to occur in the vicinity of $t=0$. In this paper we focus on the specific case $\lambda=1$; it may be shown that varying $\lambda$ does 
not alter the qualitative dynamics of the problem provided $\lambda=O(1)$. Moreover, $\lambda=O(1)$ is also a realistic assumption since, using values for lithium iron phosphate of $I_{0}=1.75 \times 10^{-2} \mathrm{~A} \mathrm{~m}^{-2}[7]$ and $\rho=1.26 \times 10^{28} \mathrm{~m}^{-3}$ [20], we find that $t_{\text {int }}$ is around 17 hours, which is comparable to a typical charging time-scale [8].

We therefore let $E(t)=1+t$ and solve the initial-value problem

(9) $\frac{\mathrm{d} c}{\mathrm{~d} t}=\frac{1}{2} \sqrt{\frac{1-c}{c}} \exp \left(\frac{t+2 c}{2 \epsilon}\right)-\frac{1}{2} \sqrt{\frac{c}{1-c}} \exp \left(-\frac{t+2 c}{2 \epsilon}\right), \quad c\left(-t_{0}\right)=c_{0}$.

We assume that $0<2 c_{0}<t_{0}$ and that $t_{0}, c_{0}$ and $1-c_{0}$ are all $O(1)$ to ensure that the initial position is within the attractor of the lower stable branch $S_{\text {I }}$. The step-by-step asymptotic analysis carried out below reveals four major stages along the dynamical path: (i) $c$ falls rapidly from its initial position to the 'empty' $S_{\mathbf{I}}$ branch; (ii) $c$ stays on $\mathrm{S}_{\text {I }}$ until $E$ approaches the saddle-node bifurcation at A; (iii) $c$ switches rapidly to the 'full' branch $\mathrm{S}_{\text {III }}$; (iv) $c$ remains on $\mathrm{S}_{\text {III }}$ as $E$ increases further. The behavior of a typical numerical solution is shown in Figure 2(b), where $C$ labels the initial point $(c, E)=\left(c_{0}, 1-t_{0}\right)=(0.25,-1)$ in this example.

(i) Initial transition to lower branch. Because the initial voltage $E$ is well below $\mu^{*}$, the concentration falls rapidly to the lower stable branch $S_{\text {I. }}$ In a tiny neighborhood of the initial time $-t_{0}$, the positive term on the right-hand side of (9) is exponentially small, while the negative term is exponentially large. Thus, we see that the leading-order dynamics is governed by

$$
\frac{\mathrm{d} c}{\mathrm{~d} t} \sim-\frac{1}{2} \mathrm{e}^{t_{0} / 2 \epsilon}\left(\frac{1-c}{c}\right)^{1 / 2} \mathrm{e}^{-c / \epsilon} .
$$

Solutions of this equation decrease exponentially quickly, with $c \rightarrow 0$ after a finite time $t+t_{0}=t_{1}$, where

$$
t_{1} \sim 2 \epsilon\left(\frac{1-c_{0}}{c_{0}}\right)^{1 / 2} \exp \left(\frac{2 c_{0}-t_{0}}{2 \epsilon}\right) .
$$

When $c=O\left(\mathrm{e}^{-t_{0} / \epsilon}\right)$, the positive term in (9) becomes important, and prevents $c$ from reaching zero. Instead, we find that the solution rapidly attains a quasiequilibrium, where the positive and negative terms in (9) balance, over a timescale where $t=-t_{0}+t_{1}+O\left(\mathrm{e}^{-t_{0} / \epsilon}\right)$. In summary, regardless of the initial conditions, $c$ undergoes an extremely rapid transition (over an exponentially small timescale) and joins the slow-varying stable branch $\mathrm{S}_{\text {. }}$.

(ii) Lower stable branch solution. Now $c$ is exponentially small and, up to exponentially small correction terms, (9) becomes

$$
\frac{\mathrm{d} c}{\mathrm{~d} t} \sim \frac{\mathrm{e}^{t / 2 \epsilon}}{2 \sqrt{c}}-\frac{\sqrt{c} \mathrm{e}^{-t / 2 \epsilon}}{2} .
$$

The asymptotic solution to (12) as $\epsilon \rightarrow 0$ takes the form

$$
c \sim \mathrm{e}^{t / \epsilon}-\frac{2}{\epsilon} \mathrm{e}^{2 t / \epsilon}+O\left(\mathrm{e}^{3 t / \epsilon}\right) \quad(t<0),
$$

which predicts slow evolution along the lower stable branch $\mathrm{S}_{1}$. Note that the solution has ceased to depend on the values of $t_{0}$ and $c_{0}$, and so the initial conditions have negligible effect on the subsequent behavior in stages (iii) and (iv). 


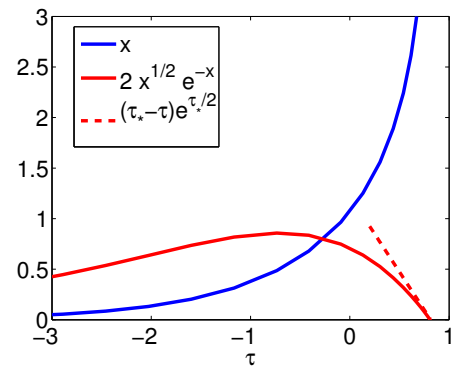

Fig. 3: Numerical solution of the initial-value problem (15). The three curves show the scaled concentration $x(\tau)$, the function $2 \sqrt{x(\tau)} \mathrm{e}^{-x(\tau)}$ and the linear fit (16).

Once $\mathrm{e}^{t / \epsilon}$ and $\epsilon$ are the same order, the two terms in (13) are balanced and hence (13) ceases to be a valid asymptotic expansion. Thus, we find that the next stage in the dynamics occurs when $t=-\epsilon \log (1 / \epsilon)+O(\epsilon)$. This is consistent with the equilibrium analysis in subsection 2.2 , where a jump in concentration at $t=-\epsilon \log (2 / \epsilon)-\epsilon$ is predicted from equation (8).

(iii) Jump solution. As $t$ approaches 0 , the positive term in (9) eventually outweighs the negative term, causing $c$ to jump from the lower to the upper stable branch. To show how $c$ exits the lower branch, we perform the scalings

$$
c=\epsilon x, \quad t=-\epsilon \log (1 / \epsilon)+\epsilon \tau .
$$

The leading-order dynamics in this regime is governed by the canonical equation

$$
\frac{\mathrm{d} x}{\mathrm{~d} \tau}=\frac{1}{2 \sqrt{x}} \mathrm{e}^{\tau / 2+x}-\frac{\sqrt{x}}{2} \mathrm{e}^{-\tau / 2-x}=\sinh \left(\frac{\tau}{2}+x-\frac{1}{2} \log (x)\right),
$$

subject to the matching condition

$$
x(\tau) \sim \mathrm{e}^{\tau}-2 \mathrm{e}^{2 \tau}+\cdots \quad \text { as } \tau \rightarrow-\infty,
$$

obtained from (13).

The numerical solution $x(\tau)$ of the initial value problem (15) is plotted in Figure 3. The solution blows up at a finite value of $\tau=\tau^{*}$, where the local behavior is

$$
2 \sqrt{x(\tau)} \mathrm{e}^{-x(\tau)} \sim \mathrm{e}^{\tau^{*} / 2}\left(\tau^{*}-\tau\right) \quad \text { as } \tau \rightarrow \tau^{*} .
$$

The dashed curve in Figure 3 demonstrates that this approximation indeed describes the behavior near $\tau=\tau^{*}$, and by fitting we find that $\tau^{*} \approx 0.81498$.

The local blow-up asymptotics (16) implies that $c=O(1)$ in the next phase, where we rescale $t$ according to

$$
t=-\epsilon \log (1 / \epsilon)+\epsilon \tau^{*}-2 \sqrt{\epsilon} \mathrm{e}^{-\tau^{*} / 2} \mathrm{e}^{-\hat{\tau} / \epsilon},
$$

where $\hat{\tau}=O(1)$. Now the positive term in (9) dominates, giving the leading order dynamics

$$
\frac{\mathrm{d} c}{\mathrm{~d} \hat{\tau}}=\sqrt{\frac{1-c}{c}} \exp \left(\frac{c-\hat{\tau}}{\epsilon}\right)
$$


up to exponentially small corrections. The solution of (18) that matches with (16) as $c \rightarrow 0$ is

$$
c=\hat{\tau}+\frac{\epsilon}{2} \log \left(\frac{\hat{\tau}}{1-\hat{\tau}}\right)+O\left(\epsilon^{2}\right) .
$$

The approximation (19) breaks down as $\hat{\tau} \rightarrow 1$, implying that $c$ increases from $O(\epsilon)$ to $1-O(\epsilon)$ exponentially rapidly as $t$ approaches $-\epsilon \log (1 / \epsilon)+\epsilon \tau^{*}$.

The remaining stages through which $c$ joins the upper stable branch are analogous to the behavior in stage (i) as it joins the lower branch. It is straightforward to show that this happens on a time scale of $t+\epsilon \log (1 / \epsilon)-\epsilon \tau^{*}=O\left(\mathrm{e}^{-1 / \epsilon}\right)$.

(iv) Upper stable branch solution. Now $c$ stays on the upper stable branch $\mathrm{S}_{\text {III }}$ as $E$ increases further, with asymptotic behavior

$$
c=1-\mathrm{e}^{-(t+2) / \epsilon}+\frac{1}{\epsilon} \mathrm{e}^{-2(t+2) / \epsilon}+O\left(\mathrm{e}^{-3(t+2) / \epsilon}\right) .
$$

Summary. Assembling the various stages, we have a complete leading-order solution to the initial value problem (9). It reveals that an effectively instantaneous transition from 'empty' to 'full' happens as $E$ passes through a critical value

$$
\mu_{\mathrm{dyn}}=1+\epsilon \tau^{*}-\epsilon \log (1 / \epsilon) .
$$

The canonical equation (15a) describes the key stage in the transition where the solution departs from the lower stable branch. A 'once and for all' numerical calculation determines the local blow-up time $\tau^{*}$, which is the only information that feeds into the global solution. Comparing (21) with the expression (8) for the equilibrium bifurcation point $\mu^{*}$, we see that dynamics delays the bifurcation by a distance

$$
\mu_{\mathrm{dyn}}-\mu^{*}=\epsilon\left(1+\log 2+\tau^{*}\right) \approx 2.51 \epsilon .
$$

This delay is universal and applies to any initial condition that is within the attractor of the lower stable branch.

\section{Development of a discrete stochastic model.}

3.1. Motivation. There are several issues arising from the deterministic model analysed above. First, it is known that thermal fluctuations may profoundly influence chemical reactions [16], but such effects are ignored in (9). The validity of this assumption should depend on the size of the nanoparticle, or equivalently the number of lithium ions each particle can host, as well as the temperature. When the number of lithium ions is relatively small, thermal fluctuations involving the addition or removal of a small number of lithium ions could have a significant impact. This indicates a second important problem to consider, namely the discreteness of the system. It remains unclear whether it is reasonable to describe the concentration using the continuous function $c(t)$, or whether there is a size limit below which we should switch from a continuum model to a discrete model.

To address both of these issues, we now develop a stochastic model under the framework of the chemical master equation. We treat the concentration $c(t)$ as a random variable in a state space; then randomness is described by its probability distribution, while the discreteness is reflected in the number of available states. The analysis of the deterministic model carried out in subsection 2.3 revealed the subtle asymptotic behavior of the delayed transition between the two equilibria. Our aim now is to determine how this delicate dynamics changes in the presence of thermal noise and discreteness. 
3.2. Chemical master equation. A natural state space to consider consists of the discrete concentration $c \in\{i \Delta C: i=1, \ldots, N\}$, where $\Delta C=1 /(N+1)$ to ensure that the completely empty and completely full states are never reached. Thus $N$ represents the number of available states, or equivalently the total number of lithium ions that each nano-particle can accommodate. Smaller $N$ means that discreteness is more likely to play a prominent role; we will show that the importance of discreteness is best measured by the product $N \epsilon$. We emphasise that we are considering here a single nanoparticle which can occupy a discrete set of $N$ states, in contrast with previous studies (for example, $[2,9]$ ) which consider a discrete set of $N$ nanoparticles.

The dynamics of lithiation is modeled by a standard one-step process [16]. Let $p_{i}(t)$ denote the probability of the system being in state $i$ at time $t$. The chemical master equation for $p_{i}$ reads

$$
\begin{aligned}
\frac{\mathrm{d} p_{i}}{\mathrm{~d} t} & =q_{i-1}^{+} p_{i-1}-q_{i}^{-} p_{i}-q_{i}^{+} p_{i}+q_{i+1}^{-} p_{i+1}, \quad i=2, \cdots, N-1 \\
\frac{\mathrm{d} p_{1}}{\mathrm{~d} t} & =-q_{1}^{+} p_{1}+q_{2}^{-} p_{2}, \\
\frac{\mathrm{d} p_{N}}{\mathrm{~d} t} & =q_{N-1}^{+} p_{N-1}-q_{N}^{-} p_{N},
\end{aligned}
$$

where $q_{i}^{ \pm}$denote the positive and negative transition probabilities from state $i$. Alternatively, (23) may be written in matrix form as

$$
\frac{\mathrm{d} \mathbf{P}}{\mathrm{d} t}=\mathbb{W} \mathbf{P}
$$

where $\mathbf{P}=\left[p_{1}, \cdots, p_{N}\right]^{T}$ and the transition matrix $\mathbb{W}$ is tridiagonal:

$$
\mathbb{W}_{i j}= \begin{cases}-\left(q_{i}^{+}+q_{i}^{-}\right) & i=j, \\ q_{i+1}^{-} & i-j=-1, \\ q_{i-1}^{+} & i-j=1, \\ 0 & \text { otherwise. }\end{cases}
$$

The transition probability $q_{i}^{+}$corresponds to system in state $i$ acquiring a single ion, and we assume that the probability of doing so depends on the free energies $g_{i}=g\left(c_{i}\right)$ and $g_{i+1}=g\left(c_{i+1}\right)$ of the two competing states, where

$$
g(c)=c(1-c)+\epsilon(c \log (c)+(1-c) \log (1-c))
$$

is the dimensionless version of (1). Similarly, we assume that $q_{i}^{-}$is a function of $g_{i}$ and $g_{i-1}$. To ensure that (23) is consistent with the Butler-Volmer dynamics (6) in the deterministic limit, we assume the specific functional forms

$$
\begin{array}{ll}
q_{i}^{+}=\frac{1}{2 \Delta C} \exp \left[\frac{1}{2 \epsilon}\left(E-\frac{g_{i+1}-g_{i}}{\Delta C}\right)\right], & i=1, \cdots, N-1, \\
q_{i}^{-}=\frac{1}{2 \Delta C} \exp \left[\frac{1}{2 \epsilon}\left(-E+\frac{g_{i}-g_{i-1}}{\Delta C}\right)\right], & i=2, \cdots, N,
\end{array}
$$

where $E$ is again the externally applied voltage. 


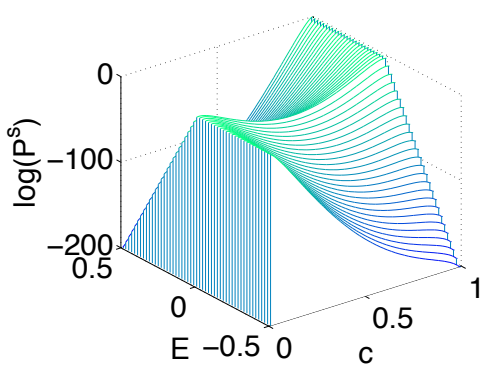

(a)

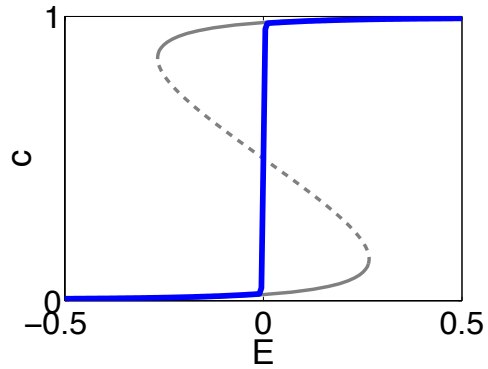

(b)

Fig. 4: Behavior of the stationary distribution (28) with $\epsilon=0.25$ and $N=100$ : (a) $\log p_{i}^{\mathrm{s}}$ plotted versus $c=i \Delta C$ and $E$; (b) mean concentration plotted versus $E$, with the equilibrium curve from Figure 2(a) for the corresponding deterministic model plotted in light gray.

3.3. Stationary distribution. The stationary distribution (denoted as $p_{i}^{\mathrm{s}}$ ) under a constant potential $E$ is given by

$$
p_{i}^{\mathrm{s}}=\exp \left[\frac{1}{\epsilon}\left((i-1) E-\frac{g_{i}-g_{1}}{\Delta C}\right)\right] p_{1}^{\mathrm{s}},
$$

where $p_{1}^{\mathrm{s}}$ is fixed by normalizing so that $\sum_{i=1}^{N} p_{i}^{\mathrm{s}}=1$. The stationary distribution $p_{i}^{\mathrm{s}}$ is plotted versus $c=i \Delta C$ and $E$ in Figure 4 with $\epsilon=0.25$ and $N=100$. If $E$ is far from 0 , the distribution is strongly localized, so that the behavior of the stochastic model appears close to deterministic. In contrast, when $E$ is near 0 , the distribution becomes bimodal, so the system is likely to be in either a 'full' or an 'empty' state. However, as shown in Figure 4(b), the average concentration transitions directly from $c \approx 0$ to $c \approx 1$ at $E \approx 0$, following the Maxwell line for phase separation [17]. The same behavior has been reported in [8] for a system of connected particles. Such bimodal behavior clearly cannot be captured by a deterministic model.

These features can also be illustrated by simulating a collection of random walks, each of which is sampled from a compound Poisson process whose transition probabilities are specified by (27), with $E$ held constant. Some results of this approach are summarized in Figure 5, where we compare realisations of such random walks, with $N=200$ and $\epsilon=0.2$, against corresponding solutions of the deterministic model (6). The values of $E$ and initial conditions are chosen purely for the sake of illustration. The stationary distribution (28), and hence the ultimate average concentration, are independent of the initial conditions imposed on the random process, although different initial conditions would affect the time taken for the stationary distribution to be achieved.

Figure 5(a) shows an example where $E$ is far from 0 and the equilibrium distribution is localized at $c \approx 1$. We show a number of random walks initialised at $i=1$, each of which is strongly attracted to the 'full' state. The average over many such random walks (solid, red) is well captured by the corresponding solution of the deterministic model (dashed, blue). In contrast, Figure 5(b) shows an example where $E$ is close to 0 , so the equilibrium distribution is bimodal and close to symmetric. Again we show a number of random walks, here initialised at $i=100$, corresponding 


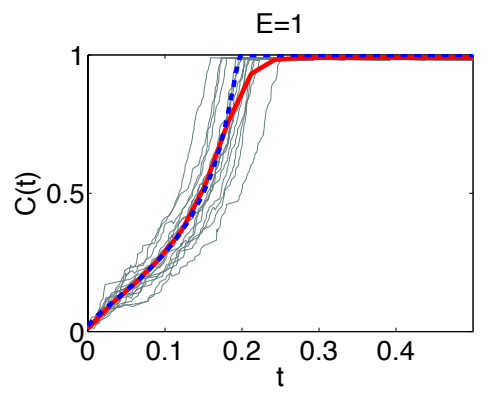

(a)

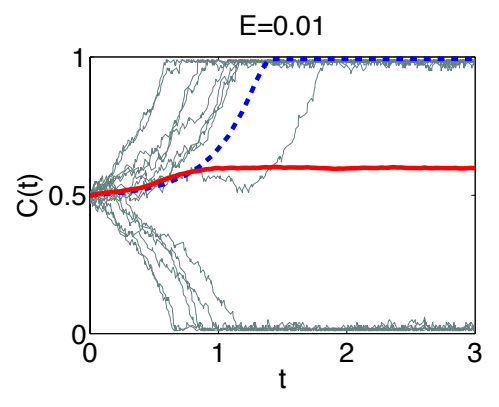

(b)

Fig. 5: The concentration evolution for a collection of random walks, with $N=200$, $\epsilon=0.2$ and (a) $E=1$; (b) $E=0.01$. Realisations of 15 random walks are shown as light gray curves; the averaged concentration over 200 such simulations is plotted as a red solid curve; and the deterministic solution is plotted as a blue dashed curve. The starting states are (a) the ground state $i=1$; (b) the middle state $i=100$.

to $c=1 / 2$. We see that some trajectories end up in the 'empty' state, while others end up in the 'full' state, and the average over many such random walks (solid, red) approaches a stationary value close to a half. In contrast, the equivalent deterministic model predicts the equilibrium concentration from this initial condition to be nearly full, as indicated by the blue dashed curve.

4. Dynamics of the stochastic model. As illustrated above, the equilibrium behaviors of the deterministic and stochastic models may be qualitatively different, particularly when $E$ is close to zero and the equilibrium distribution of the stochastic model is bimodal. However, we now wish to determine whether discrete and/or stochastic effects are equally important in dynamic situations where $E$ varies with time. We therefore study the dynamics of the stochastic model given in equations (23) and (27), again assuming that the applied potential takes the form $E(t)=1+t$ on the domain $t>-t_{0}$. Provided $t_{0}>2$ so that $E\left(-t_{0}\right)<-1$ initially, the negative transition probabilities $q_{i}^{-}$are exponentially larger than the positive transition probabilities $q_{i}^{+}$for all $i$. Therefore, regardless of the initial conditions, the system rapidly relaxes to a delta distribution at the lowest-lithium state, $i=1$, in an analogous manner to stage (i) of the deterministic dynamics analysed in subsection 2.3.

The delta distribution persists until $q_{i}^{+}$and $q_{i}^{-}$start to balance when $i$ is small, which occurs when $E(t)=1-\epsilon \log (1 / \epsilon)+O(\epsilon)$, Thus the transition behavior where the distribution spreads away from $i=1$ and eventually concentrates at $i=N$ occurs in a local regime where

$$
t=-\epsilon \log (1 / \epsilon)+\epsilon \tau
$$

and $\tau=O(1)$. This coincides with the scaling (14) for the jump stage (iii) in the deterministic model.

Following the rescaling (29), we now derive an approximation to the master equation (23) which exploits both the smallness of $\epsilon$ and the assumed largeness of $N$. We 
find that a distinguished limit occurs when $N=O(1 / \epsilon)$, and we therefore define

$$
\alpha=\frac{\Delta C}{\epsilon}=\frac{1}{(N+1) \epsilon} .
$$

As we will see, the parameter $\alpha$ measures the importance of discreteness; when $\alpha \rightarrow 0$, the model behaves mostly as a continuum, while if $\alpha \rightarrow \infty$, discrete effects are very important. These limiting cases will be dealt with in more detail in section 5 , but for now we consider the case where $\alpha=O(1)$ and we take the limit as $\epsilon \rightarrow 0$ and $N \rightarrow \infty$. As usual, the limit $N \rightarrow \infty$ results in a Fokker-Planck (F-P) type partial differential equation for a smooth function $p(c, t)$ which approximates the discrete distribution function $p_{i}(t)$. However, this continuum approximation breaks down in neighborhoods of $c=0$ and $c=1$. We therefore end up with a hybrid discrete-continuum model, which looks like a continuous $\mathrm{F}-\mathrm{P}$ equation for intermediate states, and a discrete system of master equations for boundary states.

Below we analyse in turn a discrete 'left inner layer' near $i=1$, a continuous 'outer region' where $c$ and $(1-c)$ are $O(1)$, and a discrete 'right inner layer' where $i$ is close to $N$. By matching the three regions together, we obtain a complete description of the dynamic process whereby the probability mass transitions from being concentrated at $i=1$ to concentrated at $i=N$. All of the analysis in this section will concentrate on the time domain where $\tau=O(1)$ in (29), corresponding to the key 'jump' time scale dealt with for the deterministic problem in stage (iii) of subsection 2.3.

4.1. Left inner layer. We define $\mathbf{P}^{\mathrm{L}}=\left[p_{1}, p_{2}, \cdots, p_{K}\right]^{T}$, where $K$ is an intermediate asymptotic variable such that $1 \ll K \ll N$. The left inner problem is then written as

$$
\frac{\mathrm{d} \mathbf{P}^{\mathrm{L}}}{\mathrm{d} \tau}=\mathbb{W}_{\mathrm{L}} \mathbf{P}^{\mathrm{L}},
$$

where the probability transition matrix $\mathbb{W}_{\mathrm{L}}$ is $\epsilon$ times the $K \times K$ upper-left block of the full transition matrix $\mathbb{W}$ defined by (25). The overall probability of this layer is not conserved, since summing up the rows of (31) gives

$$
\sum_{i=1}^{K} \frac{\mathrm{d} p_{i}}{\mathrm{~d} \tau}=-\epsilon q_{K}^{+} p_{K},
$$

so the left inner layer loses probability mass to the outer region at a rate $-\epsilon q_{K}^{+} p_{K}$.

After performing the rescaling (29) and letting $\epsilon \rightarrow 0$, we find that the transition probabilities $q_{i}^{+}$and $q_{i}^{-}$in this region can be simplified to

$$
q_{i}^{+} \sim \epsilon^{-1} \mathrm{e}^{\tau / 2} f_{i}^{+}(\alpha), \quad q_{i}^{-} \sim \epsilon^{-1} \mathrm{e}^{-\tau / 2} f_{i}^{-}(\alpha),
$$

where $f_{i}^{ \pm}(\alpha)$ are defined as

$$
\begin{array}{ll}
f_{i}^{+}(\alpha)=\frac{1}{2 \alpha^{3 / 2}} \exp \left(\frac{1+\alpha(2 i+1)+i \log (i)-(i+1) \log (i+1)}{2}\right), & i \geq 1 \\
f_{i}^{-}(\alpha)=\frac{1}{2 \alpha^{1 / 2}} \exp \left(\frac{-1-\alpha(2 i-1)+i \log (i)-(i-1) \log (i-1)}{2}\right), & i \geq 2
\end{array}
$$

Therefore the transition matrix $\mathbb{W}_{\mathrm{L}}$ can be decomposed into two components: a forward term that drives the probability density to higher states and a backward term which drives the probability density towards lower states:

$$
\mathbb{W}_{\mathrm{L}}=\mathrm{e}^{\tau / 2} F^{+}(\alpha)+\mathrm{e}^{-\tau / 2} F^{-}(\alpha)
$$



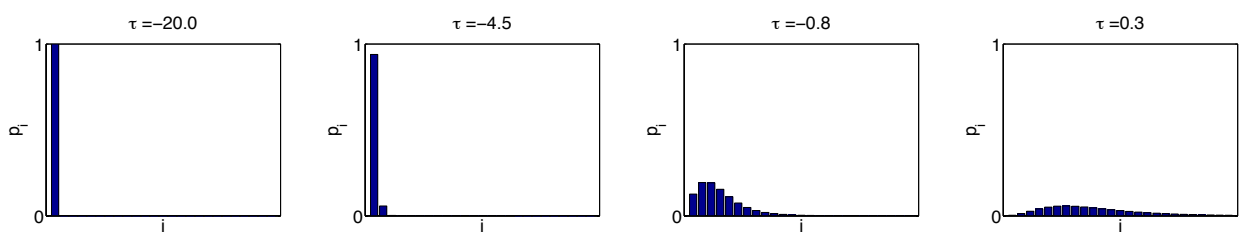

Fig. 6: Simulation of the left inner layer problem (37) with $\alpha=0.2$ and $K=25$.
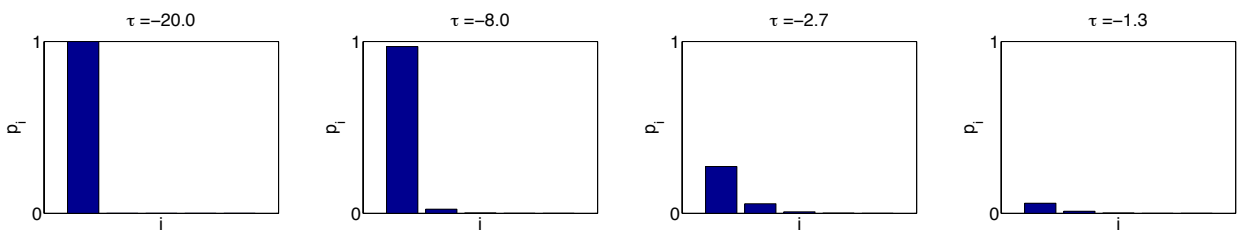

Fig. 7: Simulation of the left inner layer problem (37) with $\alpha=2$ and $K=5$.

where $F^{+}(\alpha)$ and $F^{-}(\alpha)$ are two constant tridiagonal matrices given by

$$
F_{i j}^{+}=\left\{\begin{array}{ll}
-f_{i}^{+}(\alpha) & i=j, \\
f_{i-1}^{+}(\alpha) & i=j+1, \\
0 & \text { otherwise, }
\end{array} \quad F_{i j}^{-}= \begin{cases}-f_{i}^{-}(\alpha) & i=j, \\
f_{i+1}^{-}(\alpha) & i=j-1, \\
0 & \text { otherwise. }\end{cases}\right.
$$

The left inner layer master equation (31) therefore reduces to

$$
\frac{\mathrm{d} \mathbf{P}^{\mathrm{L}}}{\mathrm{d} \tau}=\mathrm{e}^{\tau / 2} F^{+}(\alpha) \mathbf{P}^{\mathrm{L}}+\mathrm{e}^{-\tau / 2} F^{-}(\alpha) \mathbf{P}^{\mathrm{L}} .
$$

When $\tau$ is large and negative, the negative flux term $F^{-}$dominates and drives the system towards a delta distribution at $i=1$. As $\tau$ passes through zero, the positive and negative fluxes balance and the distribution begins to spread away from $i=1$. As $\tau$ increases further, the probability is driven towards ever higher states, and the total probability mass remaining in the left inner layer decreases towards zero.

The flux into the left inner layer from the outer region is negligible throughout, because the product $F_{i K}^{-} p_{K}$ is always exponentially small when $K \gg 1$. Therefore, the inner layer problem (37) may be simulated independently of the outer problem, subject to the initial condition

$$
p_{i} \rightarrow \delta_{1 i}, \quad \text { as } \tau \rightarrow-\infty .
$$

We find that solutions generically exhibit one of two distinctive patterns. At small values of $\alpha$, the initial delta distribution spread out and travels towards higher states as a wave, as shown in Figure 6 (with $\alpha=0.2$ ). In contrast, for larger values of $\alpha$, the distribution remains highly localized at $i=1$ for all $\tau$, as shown in Figure 7 (with $\alpha=2$ ). This qualitative change in behavior will be explored further in section 5 by examining the asymptotic limits where $\alpha \ll 1$ and $\alpha \gg 1$. In either case, the overall probability of the left inner layer eventually decreases as a result of the flux into the outer layer. 


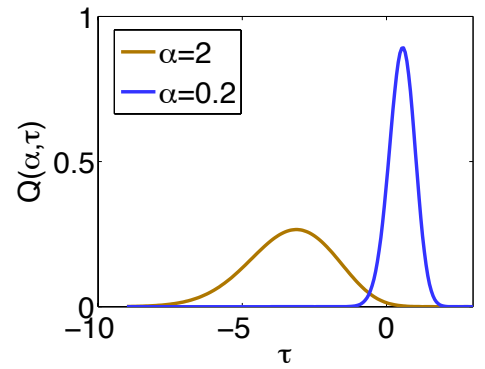

(a)

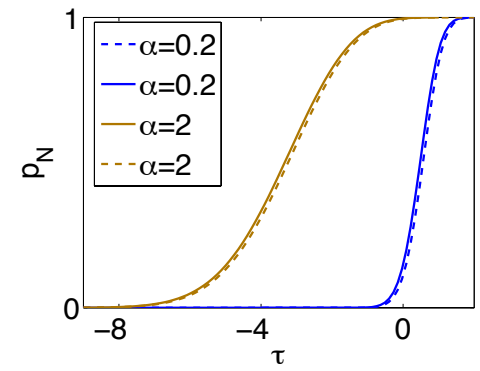

(b)

Fig. 8: (a) The numerically determined function $Q(\alpha ; \tau)$ and (b) the probability density $p_{N}(\tau)$ of the highest state, plotted versus $\tau$ with $\alpha=2$ and $\alpha=0.2$. The solid curves show the solution of the approximate equation (45), and the dashed curves show the results of solving the full master equation (23).

To obtain the solution in the outer region which matches the behavior of the inner layer, we require the limiting behavior of $p_{i}(\tau)$ from the inner layer model as $i \rightarrow \infty$. For large $i$, the positive flux term in (37) dominates, and hence we find that

$$
\mathrm{e}^{\tau / 2} p_{i}(\tau) f_{i}^{+}(\alpha) \rightarrow Q(\alpha ; \tau) \quad \text { as } i \rightarrow \infty .
$$

Here the function $Q$, which measures the net probability flux out of the inner layer, is independent of $i$, and is determined in principle by numerical solution of the discrete inner problem (37) and (38).

We plot $Q(\alpha ; \tau)$ versus $\tau$ in Figure $8($ a) for the cases $\alpha=0.2$ and $\alpha=2$, corresponding to the distributions plotted in Figures 6 and 7 respectively. Generically $Q$ increases to a maximum and then decreases again as a function of $\tau$, as the distribution function initially concentrated at $i=1$ spreads and propagates out of the inner layer. For the smaller value of $\alpha$, the peak in $Q$ is both higher and narrower, and the time taken for the probability mass to leave the inner region completely is longer. Each graph has unit area, by net conservation of probability.

4.2. Outer problem. In the outer region, where $i \Delta C=O(1)$, the probability distribution is approximately described by a continuous function $p_{i}(\tau) \sim p(c, \tau)$, where $c=i \Delta C \in(0,1)$. Under the scaling (29), the leading-order transition probabilities in the outer layer are given by

$$
\begin{aligned}
& q_{i}^{+} \sim q^{+}(c, \tau)=\frac{\mathrm{e}^{(\alpha+\tau) / 2}}{2 \alpha \epsilon^{1 / 2}} \sqrt{\frac{1-c}{c}} \mathrm{e}^{c / \epsilon}, \\
& q_{i}^{-} \sim q^{-}(c, \tau)=\frac{\mathrm{e}^{(\alpha-\tau) / 2}}{2 \alpha \epsilon^{3 / 2}} \sqrt{\frac{c}{1-c}} \mathrm{e}^{-c / \epsilon} .
\end{aligned}
$$

Hence the positive term $q^{+}$dominates $q^{-}$, and the $\mathrm{F}-\mathrm{P}$ equation reduces to

$$
\frac{\partial p}{\partial \tau} \sim-\frac{\epsilon^{1 / 2} \mathrm{e}^{(\alpha+\tau) / 2}}{2 \alpha} \frac{\partial}{\partial c}\left(\mathrm{e}^{c / \epsilon} p \sqrt{\frac{1-c}{c}}\right) .
$$


Since the right-hand side is exponentially large, the outer problem is quasi-steady, and to lowest order the bracketed term is a function of $\tau$ alone. This function of integration may be determined by matching with the left inner layer via the matching condition (39), and hence we obtain the leading-order outer solution

$$
p(c, \tau)=2 \alpha Q(\alpha ; \tau) \mathrm{e}^{-(\alpha+\tau) / 2} \epsilon^{1 / 2} \mathrm{e}^{-c / \epsilon} \sqrt{\frac{c}{1-c}} .
$$

It follows that the probability for the system to be in an intermediate state, with $1 \ll i \ll N$, is exponentially small.

4.3. Right inner layer. In the right inner layer, we set $i=N+1-j$, where $j=O(1)$, and find that the leading-order transition probabilities are given by

$$
q_{N+1-j}^{+} \sim \mathrm{e}^{1 / \epsilon} \mathrm{e}^{\tau / 2} f_{j}^{-}(\alpha), \quad q_{N+1-j}^{-} \sim \epsilon^{-2} \mathrm{e}^{-1 / \epsilon} \mathrm{e}^{-\tau / 2} f_{j}^{+}(\alpha),
$$

where the functions $f^{ \pm}(\alpha)$ are still defined by equation (34). Since $q_{N+1-j}^{+}$is exponentially large, the right inner layer is also quasi-steady to leading order, with $q_{N+1-j}^{+} p_{N+1-j}$ independent of $j$ and

$$
q_{N-1}^{+} p_{N-1}=q_{N-2}^{+} p_{N-2}=q_{N-3}^{+} p_{N-3}=\cdots=\frac{\mathrm{d} p_{N}}{\mathrm{~d} \tau} .
$$

On the other hand, matching with the outer solution (42) gives $q_{N+1-j}^{+} p_{N+1-j} \rightarrow Q$ as $j \rightarrow \infty$ and hence

$$
\frac{\mathrm{d} p_{N}}{\mathrm{~d} \tau}=Q(\alpha ; \tau)
$$

In conclusion, the probability flux out of the inner layer is effectively transferred directly to the highest state $i=N$, with the probabilities of all intermediate states remaining exponentially small throughout. The resulting prediction for the probability density $p_{N}(\tau)$ of the highest state is plotted in Figure $8(\mathrm{~b})$ for the same values $\alpha=2$ and $\alpha=0.2$ as above. We see that the dynamics of the full master equation system (23) is captured extremely well by the approximate equation (45).

5. Discrete and continuum limits of the stochastic model. We have shown in the previous section that the transition of the probability mass from the lowestlithium state $i=1$ to the highest-lithium state $i=N$ is described by the solution of the canonical discrete system (37) and (38) in the left inner region. In general this problem must be solved numerically for each value of $\alpha=((N+1) \epsilon)^{-1}$ to determine the flux of probability out of the inner region and thence into the highest-lithium state, $i=N$. However, we will now show how the problem may be simplified when $\alpha$ is either very small or very large. The analysis enables us to explain the qualitative behavior observed in the numerical solutions shown in Figures 6 to 8 and also shows how the effects of randomness and of discreteness in this problem are captured by the single parameter $\alpha$.

5.1. The discrete limit $\alpha \gg 1$. If the number $N$ of available states is relatively small compared with $1 / \epsilon$, then discreteness of the system governs the dynamics. In this case $\alpha \gg 1$ and it follows that $f_{i}^{+} \gg f_{i+1}^{+}$and $f_{i}^{+} \gg f_{i}^{-}$for any $i$ in the left inner layer. Hence it follows that $p_{i}(\tau) \ll p_{1}(\tau)$ for all $i \geq 1$ in the inner layer and, to lowest 


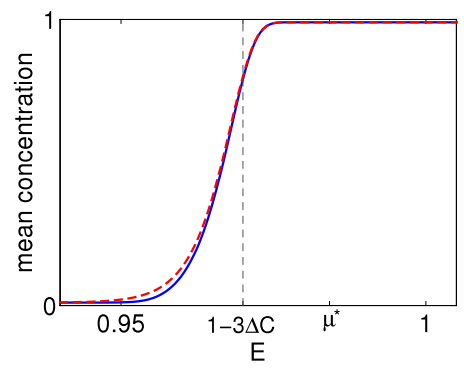

Fig. 9: Expected concentration $\bar{c}$ plotted versus potential $E$. The blue solid curve shows the analytical approximation (47) with $\alpha=5$; the red dashed curve shows the numerical simulation of the full problem (37) with $\epsilon=0.002$ and $N=99$. The static transition potential $\mu^{*}$ predicted by the deterministic model is also marked.

order, the system is always either in the lowest-lithium state or the highest-lithium state. Thus we obtain the leading-order solution

$$
p_{1}=\exp \left(-2 f_{1}^{+} \mathrm{e}^{\tau / 2}\right), \quad p_{N}=1-\exp \left(-2 f_{1}^{+} \mathrm{e}^{\tau / 2}\right),
$$

and $p_{i} \sim 0$ for $1<i<N$, where

$$
f_{1}^{+}=\frac{\mathrm{e}^{(3 \alpha+1) / 2}}{4 \alpha^{3 / 2}} .
$$

We infer from (46) that the dynamic transition of $p_{1}$ from 1 to 0 (while $p_{N}$ does the reverse) occurs in a neighborhood of $\tau \sim-3 \alpha$. In terms of the potential, this corresponds to $E \sim 1-3 \Delta C$, which is lower than both the equilibrium turning point $\mu^{*}$ and the dynamic transition potential $\mu_{\mathrm{dyn}}$ predicted by the deterministic model in subsection 2.3. Therefore, when discreteness dominates, with high probability the system always exists in either the $i=1$ state or the $i=N$ state, and its jump from empty to full occurs earlier than predicted by the deterministic model.

The expected concentration in this limit is approximated by

$$
\bar{c}=\frac{1}{N+1} \sum_{i=1}^{N} i p_{i}(\tau) \sim(1-\Delta C)+(2 \Delta C-1) \exp \left(-\frac{\mathrm{e}^{(3 \alpha+1+\tau) / 2}}{2 \alpha^{3 / 2}}\right) .
$$

In Figure 9, we show the excellent agreement between the approximation (47) and the mean concentration obtained by solving the chemical master equation (23) for a case where $\alpha=5$. We observe that the jump from 'empty' to 'full' occurs close to the predicted value $E=1-3 \Delta C=0.97$, which is noticeably lower than the static prediction $\mu^{*} \approx 0.984$.

5.2. The continuum limit $\alpha \ll 1$. At the opposite extreme where $\alpha \ll 1$, the number of available states is sufficiently large compared with $1 / \epsilon$ for even the 'left inner' problem to be treated using a continuum model. As $\alpha \rightarrow 0$, the master equation (37) reduces to

$$
\alpha \frac{\mathrm{d} p_{i}}{\mathrm{~d} \tau^{\prime}}=\mathrm{e}^{\tau^{\prime} / 2}\left(\tilde{f}_{i-1}^{+} p_{i-1}-\tilde{f}_{i}^{+} p_{i}\right)+\mathrm{e}^{-\tau^{\prime} / 2}\left(\tilde{f}_{i+1}^{-} p_{i+1}-\tilde{f}_{i}^{-} p_{i}\right),
$$



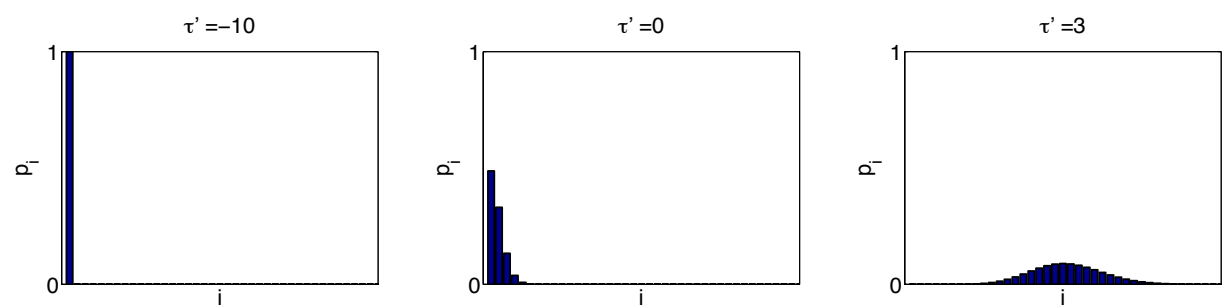

Fig. 10: Quasi-equilibrium distribution prescribed by (50) with the sum $\sum p_{i}$ normalized to 1 .

where $\tau^{\prime}=\tau-\log \alpha$ and the coefficients

$$
\begin{array}{ll}
\tilde{f}_{i}^{+}=\frac{1}{2} \exp \left(\frac{1+i \log (i)-(i+1) \log (i+1)}{2}\right), & i \geq 1, \\
\tilde{f}_{i}^{-}=\frac{1}{2} \exp \left(\frac{-1+i \log (i)-(i-1) \log (i-1)}{2}\right), & i \geq 2
\end{array}
$$

no longer depend on $\alpha$.

To leading order in $\alpha$, the system is in quasi-equilibrium, with distribution given by

$$
p_{i}\left(\tau^{\prime}\right) \propto \mathrm{e}^{(i-1) \tau^{\prime}} \mathrm{e}^{i-1-i \log i}
$$

up to a normalization factor. This distribution is plotted in Figure 10 to reveal how it gradually changes from a delta distribution to a Gaussian as $\tau^{\prime}$ increases. As $\tau^{\prime}$ and $i$ tend to infinity, one can easily verify that (50) does indeed approach a Gaussian, namely

$$
p_{i}(\tau) \sim \frac{\mathrm{e}^{-\tau^{\prime} / 2}}{\sqrt{2 \pi}} \exp \left(\frac{\left(i \mathrm{e}^{-\tau^{\prime} / 2}-\mathrm{e}^{\tau^{\prime} / 2}\right)^{2}}{2}\right)=\frac{\sqrt{\alpha}}{\sqrt{2 \pi}} \mathrm{e}^{-\tau / 2} \mathrm{e}^{\mathrm{e}^{-\tau} \xi^{2} / 2}
$$

where

$$
\xi=\frac{i \alpha-\mathrm{e}^{\tau}}{\sqrt{\alpha}}
$$

In the leading-order equations (48) and (49), we have neglected terms of order $\alpha i$. Hence the approximate solution (50) ceases to be valid for large values of $\tau^{\prime}$ when the tail of the distribution has spread as far as $i=O(1 / \alpha)$. In this later stage of the evolution, we define $x=i \alpha$ and $p(x, \tau)=p_{i}(\tau)$, and find that the approximately continuous distribution is now described by the partial differential equation

$$
\frac{\partial p}{\partial \tau}=-\frac{\partial}{\partial x}(p \sinh (\tau / 2+x-\log x / 2))+\frac{\alpha}{2} \frac{\partial^{2}}{\partial x^{2}}(p \sinh (\tau / 2+x-\log x / 2)) .
$$

The $\mathrm{F}-\mathrm{P}$ equation (53) admits solutions of Gaussian form

$$
p(x, \tau)=\sqrt{\alpha} A(\tau) \exp \left(-\pi A(\tau)^{2} \xi^{2}\right), \quad \text { where } \quad \xi=\frac{x-\bar{x}(\tau)}{\sqrt{\alpha}} .
$$



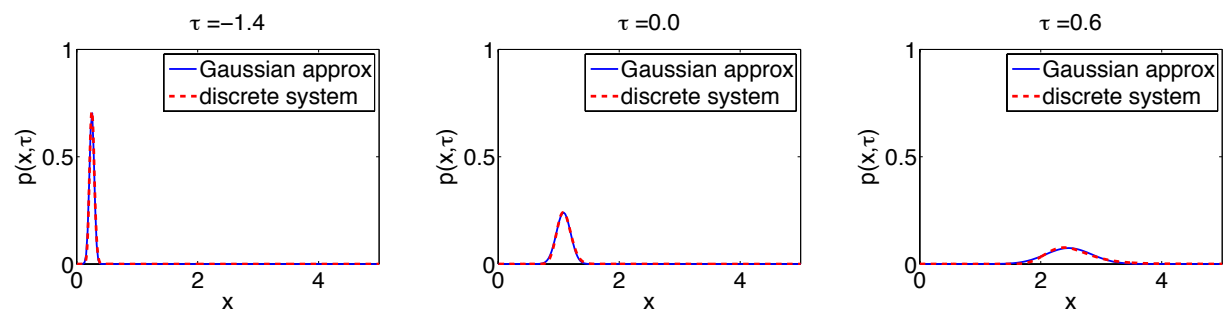

Fig. 11: Distribution profiles computed by using the continuum approximation (54) and by the solution of the discrete system (37) with $\alpha=5 \times 10^{-3}$ and $K=10^{3}$.

The distribution is then fully determined by its mean $\bar{x}(\tau)$ and amplitude $A(\tau)$, whose dynamics we can find by plugging the ansatz (54) into (53), resulting in

$$
\begin{aligned}
\frac{\mathrm{d} \bar{x}}{\mathrm{~d} \tau} & =\sinh \left(\frac{\tau}{2}+\bar{x}-\frac{1}{2} \log \bar{x}\right), \\
\frac{\mathrm{d} A}{\mathrm{~d} \tau} & =\frac{1}{2}\left(-1+\frac{1}{2 \bar{x}}-\pi A^{2}\right) A \cosh \left(\frac{\tau}{2}+\bar{x}-\frac{1}{2} \log \bar{x}\right) .
\end{aligned}
$$

The initial conditions are found by matching with (51):

$$
\bar{x}(\tau) \sim \mathrm{e}^{\tau}, \quad A(\tau) \sim \frac{\mathrm{e}^{-\tau / 2}}{\sqrt{2 \pi}} \quad \text { as } \tau \rightarrow-\infty,
$$

i.e. the discrete initial dynamics selects a specific trajectory for the ODE system (55).

Now we see that the mean concentration $\bar{x}(\tau)$ follows the same initial-value problem (15) as predicted by the deterministic model near transition from the lower to the upper equilibrium branch. From the analysis of subsection 2.3, we know that $\bar{x}(\tau)$ tends to infinity as $\tau \rightarrow \tau^{*} \approx 0.81498$; at the same time $A(\tau)$ approaches zero, with

$$
A \sim \text { const } \sqrt{\tau-\tau^{*}} .
$$

Therefore, as $\tau \rightarrow \tau^{*}$, the probability mass flows completely out of the left inner region and accumulates effectively instantaneously at the highest state $i=N$, as described in subsection 4.3 .

To check the conclusions of this Section, we solve (55) numerically subject to (56), and compare with the corresponding numerical solutions of the chemical master equation (37) with $\alpha=5 \times 10^{-3}$ and $K=10^{3}$. In Figure 11, we compare the predicted distribution functions at three different values of $\tau$. In Figures 12(a) and 12(b), we compare the mean $\bar{x}(\tau)$ and amplitude $A(\tau)$. Finally, in Figure 12(c) we compare the average concentration $\bar{c}(\tau)=\epsilon \bar{x}(\tau)$, as predicted both by the continuum limiting problem (55) and (56) and by the deterministic 'jump' problem (15), with the average over 200 random walks simulated using the transition probabilities (27). The agreement is in all cases excellent, at least until the predicted mean concentration $\bar{x}$ blows up as $\tau \rightarrow \tau^{*}$.

6. Conclusions. In this paper, we develop mathematical models for the lithiation/delithiation process of a single cathode nanoparticle in a lithium battery. We examine the process from two different perspectives. 


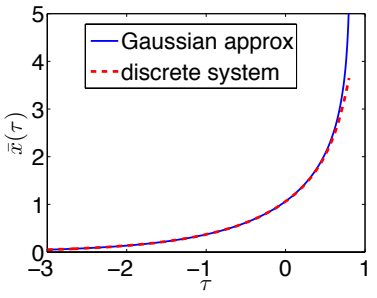

(a)

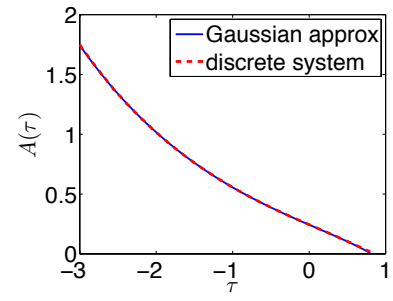

(b)

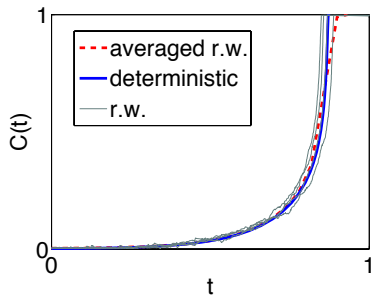

(c)

Fig. 12: The left two panels show comparisons of (a) the distribution mean $\bar{x}$ and (b) the amplitude $A$ predicted by the continuum approximation (55) and (56) and by the discrete system (37) with $\alpha=5 \times 10^{-3}$ and $K=10^{3}$. (c) The average concentration $\bar{c}(t)$ predicted by both continuum and discrete models as well as by averaging over many random walks; here the parameter values are $\epsilon=0.025$ and $N=399$, so that $\alpha=0.1$.

First, we take the approach of representing the lithium concentration in a particle by a continuum variable that behaves as a deterministic function of time. In the physically relevant limit where $\epsilon=k_{\mathrm{B}} T / \Omega \ll 1$, so it is energetically very favorable for the particle to be either 'empty' or 'full', the governing ODE (15) is stiff, and its solutions exhibit slow quasi-equilibrium dynamics with fast transitions between equilibria. We analyse the dynamics of this model using perturbation methods, and identify a critical voltage where the nanoparticle suddenly jumps from 'empty' to 'full' as the voltage is increased. This critical dynamic voltage $\mu_{\mathrm{dyn}}$ is unconditionally larger than the corresponding static bifurcation value $\mu^{*}$.

To determine whether this solution structure is robust to discrete effects and thermal fluctuations, we also consider a stochastic model for the probability distribution of the lithium concentration in a nanoparticle, assuming that the concentration can vary over a discrete state space of size $N$. We start with the chemical master equation, and again exploit the assumed smallness of $\epsilon$ to reformulate it as a continuous Fokker-Planck PDE coupled to two discrete inner layers. We show how the probability distribution evolves from a delta distribution at the lowest-lithium state to a delta distribution at the highest-lithium state as the particle is lithiated, with the probability of all intermediate states remaining small throughout the transition. The detailed shape of the probability distribution during the transition is found to depend on a single parameter $\alpha=1 /((N+1) \epsilon)$, which measures the importance of discreteness.

In the limit $\alpha \ll 1$, the probability density is approximately a continuous Gaussian, and the macroscopic law for the mean concentration is essentially identical to the deterministic model dynamics. In this case, random effects play a significant role only during a very short period: the distribution function briefly spreads out while the probability mass transfers from the ground state to higher states; thereafter it rapidly accumulates at the highest state $i=N$ and the behavior again becomes deterministic.

In the opposite limit $\alpha \gg 1$, the probability distribution is essentially degenerate, and the probability mass leaks directly from the lowest-lithium state to the highestlithium state. In this case the dynamics is not well captured by the deterministic 
model. In particular the jump from 'empty' to 'full' occurs significantly earlier than either the static $\mu^{*}$ or the dynamic $\mu_{\mathrm{dyn}}$ transition points predicted by the deterministic model.

Assuming the density of delithiated LFP is around $1.26 \times 10^{28} \mathrm{~m}^{-3}$, a particle with $10 \mathrm{~nm}$ diameter can host approximately 12000 ions [7], while values of $\epsilon$ in the range $0.2-0.4$ are reported in the literature (for example, $[6,14,21]$ ). These values suggest that a typical LFP nano-particle with diameter of 50-1000 nm can accommodate enough lithium atoms to be in the continuum regime. Our analysis therefore implies that evolution of the mean concentration during lithiation on a time-scale of order $t_{\text {int }}$ should follow the deterministic Butler-Volmer model. We note, however, that the equilibrium behavior of the stochastic model is very different from the equilibrium behavior of the deterministic model; in particular, Figure 4(b) shows that the deterministic model predicts hysteresis under quasi-static conditions, while the continuum stochastic model does not. These observations suggest that significant deviations from the deterministic model might be seen if lithiation is performed more slowly.

Our stochastic model describes the time evolution of the probability distribution over a large number of independent lithiation experiments. It could equally be used to predict the average behavior of an ensemble of completely decoupled nanoparticles. In a real cathode material, however, each nanoparticle is strongly coupled to its neighbors [9]. In principle, this complication could be introduced to either the stochastic model or the deterministic model by incorporating an interaction potential into the free energy (1). In the deterministic setting, one ends up with a coupled system of ButlerVolmer type ODEs similar to (6), but with a bifurcation structure and dynamic behavior much more complicated and delicate than the one-particle case considered in section 2. This makes it even more likely that the dynamics could be significantly influenced by stochastic effects and the deterministic model may fail to be accurate. In contrast, the full stochastic model for a coupled system of $M$ particles would in general consist of $N M$ coupled ODEs or, in the continuous limit, an $M$-dimensional FP type PDE. In either case, the full system would be very difficult to solve in general. It is hoped that the asymptotic approaches developed in this paper may be extended to derive simplified multi-particle models that capture the essential dynamics of an electrode, but which are analytically and computationally tractable.

\section{Appendix A. Non-constant exchange current.}

Here we discuss the effects of relaxing our assumption in subsection 2.1 that the exchange current $I_{0}$ is constant. As an example, consider the simple but widely used model [4] $I_{0}(c) \propto c^{1 / 2}(1-c)^{1 / 2}$, so the dimensionless deterministic model (6) becomes

$$
\frac{\mathrm{d} c}{\mathrm{~d} t}=\frac{1-c}{2} \exp \left(\frac{E-1+2 c}{2 \epsilon}\right)-\frac{c}{2} \exp \left(\frac{-E+1-2 c}{2 \epsilon}\right) .
$$

The equilibria are the same as in subsection 2.2, and we still expect the out-ofequilibrium dynamics to experience slow evolution along the stable branches and exponentially fast transitions between them as in subsection 2.3.

The major difference from the constant $I_{0}$ model occurs in the jump regime in which $E-1=O(\epsilon)$ and $c=O(\epsilon)$. In this regime the positive term on the right-hand side of (58) dominates the negative term, so the local jump dynamics is analytically solvable, unlike subsection 2.3, where a numerical solution was required. It can be shown that in this case the jump happens when $t=o(\epsilon)$.

More generally, suppose that $I_{0} \propto c^{k}(1-c)^{1-k}$. Simple calculations reveal that 


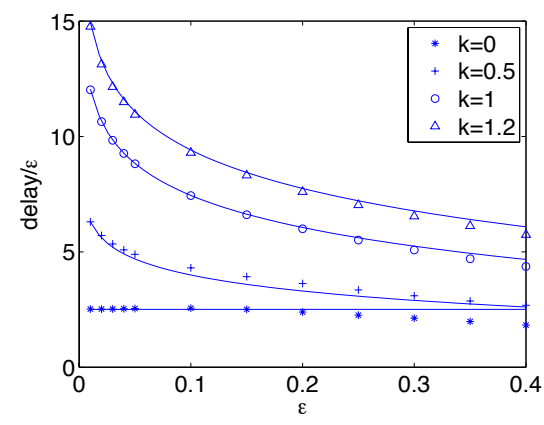

(a)

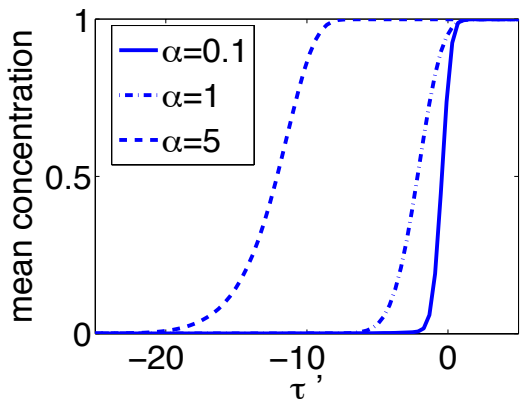

(b)

Fig. 13: (a) The scaled transition delay $\left(\mu_{\mathrm{dyn}}-\mu^{*}\right) / \epsilon$ plotted versus $\epsilon$ for different values of $k$. The solid curves show the prediction (59) of the asymptotic analysis. The markers show values obtained by solving the corresponding initial-value problem numerically, where the transition time is identified as the time when $c$ reaches $1 / 2$. (b) Mean concentration evolution when $I_{0}$ is proportional to $c^{1 / 2}(1-c)^{1 / 2}$, with the distribution in the left-inner layer determined by (61).

we need $k \geq 1 / 2$ to have the positive flux dominate in the jump regime as above. If we further specify $1 / 2 \leq k \leq 3 / 2$, then the delay in the dynamic transition time can be expressed as

$$
\frac{\mu_{\mathrm{dyn}}-\mu^{*}}{\epsilon}=2 k \log (1 / \epsilon)+1+2 \log (\Gamma(3 / 2-k))+\log 2
$$

where $\Gamma$ is the Gamma function. This should be compared with the constant- $I_{0}$ prediction (22).

We plot the prediction (59) of the delay versus numerical results under different values of $k$ in Figure 13(a). The asymptotics agree well with the numerics for $\epsilon \lesssim 0.1$ and do not deviate much for larger values of $\epsilon$. In general, a larger value of $k$ induces a longer delay, as expected physically since increasing $k$ decreases the forward current around $E \approx 1$ and therefore postpones the transition.

When applying $I_{0} \propto c^{1 / 2}(1-c)^{1 / 2}$ to the stochastic model, we find that the critical transition region in the left inner layer is $t=\epsilon t^{\prime}$, with the dynamics dominated by the positive flux term:

$$
\frac{\mathrm{d} \mathbf{P}^{\mathrm{L}}}{\mathrm{d} t^{\prime}}=\mathrm{e}^{t^{\prime} / 2} \bar{F}^{+}(\alpha) \mathbf{P}^{\mathrm{L}}+O(\epsilon)
$$

Here $\bar{F}^{+}(\alpha)$ has the same structure as $F^{+}(\alpha)$ defined by (36), and the elements are related by $\bar{f}_{i}^{+}=(\alpha i)^{1 / 2} f_{i}^{+}$. Again the problem in the transition regime is simplified, 
and there is a full analytical solution, namely

$$
\begin{aligned}
p_{1}= & \mathrm{e}^{-2 \bar{f}_{1}^{+} \mathrm{e}^{t^{\prime} / 2}}, \\
p_{2}= & \frac{\bar{f}_{1}^{+}}{\bar{f}_{2}^{+}-\bar{f}_{1}^{+}}\left(\mathrm{e}^{-2 \bar{f}_{1}^{+} \mathrm{e}^{t^{\prime} / 2}}-\mathrm{e}^{-2 \bar{f}_{2}^{+} \mathrm{e}^{t^{\prime} / 2}}\right), \\
& \vdots \\
p_{K}= & \sum_{i=1}^{K} \frac{\prod_{j=1}^{K-1} \bar{f}_{j}^{+}}{\prod_{j=1, j \neq i}^{K}\left(\bar{f}_{j}^{+}-\bar{f}_{i}^{+}\right)} \mathrm{e}^{-2 \bar{f}_{i}^{+} \mathrm{e}^{t^{\prime} / 2}} .
\end{aligned}
$$

Now all the physical quantities of interest are fully determined regardless of the value of $\alpha$. We plot the evolution of the mean concentration under three values of $\alpha$ in Figure 13(b). As in the constant- $I_{0}$ case, we observe that the greater the value of $\alpha$, the earlier the stochastic jump starts than the deterministic prediction.

\section{REFERENCES}

[1] P. Bai, D. A. Cogswell, And M. Z. Bazant, Suppression of phase transformations in $\mathrm{LiFePO}_{4}$ during battery discharge, Nano Lett., 11 (2011), pp. 4890-4896.

[2] P. Bai And G. Tian, Statistical kinetics of phase-transforming nanoparticles in lifepo4 porous electrodes, Electrochimica Acta, 89 (2013), pp. 644-651.

[3] M. Z. BAZANT, Theory of chemical kinetics and charge transfer based on nonequilibrium thermodynamics, Acc. Chem. Res., 46 (5) (2013), p. 11441160.

[4] J. O. Bockris, A. K. N. Reddy, and M. Gamboa-Aldeco, Modern Electrochemistry $2 A$. Fundamentals of Electrodics, Kluwer Academic/Plenum Publishers, 2000.

[5] D. Burch, Intercalation Dynamics in Lithium-Ion Batteries, PhD thesis, Massachusetts Institute of Technology, Sept. 2009.

[6] D. Burch And M. Z. Bazant, Size-dependent spinodal and miscibility gaps for intercalation in nanoparticles, Nano Letters, 9 (2009), pp. 3795-3800.

[7] S. Dargaville and T. W. Farrell, Predicting active material utilization in $\mathrm{LiFePO}_{4}$ electrodes using a multiscale mathematical model, J. Electrochem. Soc., 157 (2010), pp. A830A840.

[8] W. Dreyer, C. Guhlke, And M. Herrmann, Hysteresis and phase transition in many-particle storage systems, Continuum Mech. Thermodyn., 23 (2011), pp. 211-231.

[9] W. Dreyer, C. Guhlke, And R. Huth, The behavior of a many-particle electrode in a lithiumion battery, Physica D, 240 (2011), pp. 1008-1019.

[10] W. Dreyer, J. Jamnik, C. Guhlke, R. Huth, J. MošKon, and M. Gaberšček, The thermodynamic origin of hysteresis in insertion batteries, Nat. Mater., 9 (2010), p. 448453.

[11] H. Fei, Z. Peng, Y. Yang, L. Li, A.-R. Raji, E. Samuel, and J. Tour, $\mathrm{LiFePO}_{4}$ nanoparticles encapsulated in graphene nanoshells for high-performance lithium-ion battery cathodes, Chem. Commun., 50 (2014), p. 7117.

[12] T. R. FERGUSON, Lithium-ion battery modeling using non-equilibrium thermodynamics, $\mathrm{PhD}$ thesis, Massachusetts Institute of Technology, 2014.

[13] P. Gibot, M. Casas-Cabanas, L. Laffont, S. Levasseur, P. Carlach, S. Hamelet, J. TARASCON, AND C. MASQUELIER, Room-temperature single-phase Li insertion/extraction in nanoscale $\mathrm{Li}_{x} \mathrm{FePO}_{4}$, Nature Materials, 7 (2008), pp. 741-747.

[14] B. Han, A. V. Der Ven, D. Morgan, ANd G. Ceder, Electrochemical modeling of intercalation processes with phase field models, Electrochimica Acta, 49 (2004), pp. 4691 - 4699.

[15] H. Huang, S.-C. Yin, And L. F. NAZAR, Approaching theoretical capacity of $\mathrm{LiFePO}_{4}$ at room temperature at high rates, Electrochem. Solid-State Lett., 4(10) (2001), pp. A170-A172.

[16] N. V. Kampen, Stochastic Processes in Physics and Chemistry, Third Edition, North-Holland Personal Library, 2007.

[17] M. LANDSTORFer And T. JACOB, Mathematical modeling of intercalation batteries at the cell level and beyond, Chem. Soc. Rev., 42 (2013), p. 3234.

[18] R. Malik, F. Zhou, And G. Ceder, Kinetics of non-equilibrium lithium incorporation in $\mathrm{LiFePO}_{4}$, Nat. Mater., 10 (2011), p. 587590.

[19] R. Mukherjeea, R. Krishnanb, T. Luc, and N. Koratkara, Nanostructured electrodes for high-power lithium ion batteries, Nano Energy, 1 (2012), p. 518533. 
[20] B. Orvananos, T. R. Ferguson, H.-C. Yu, M. Z. Bazant, and K. Thornton, Particlelevel modeling of the charge-discharge behavior of nanoparticulate phase-separating $\mathrm{Li}$-ion battery electrodes, arXiv:1309.6495, (2013).

[21] G. K. Singh, G. Ceder, AND M. Z. BAZAnt, Intercalation dynamics in rechargeable battery materials: General theory and phase-transformation waves in $\mathrm{LiFePO}_{4}$, Electrochimica Acta, 53 (2008), pp. 7599-7613.

[22] V. SRinivasan and J. Newman, Discharge model for the Lithium Iron-Phosphate electrode, Electrode J. Electrochem. Soc., 151 (2004), pp. A1517-A1529.

[23] M. Tang, H. Huang, N. Meethong, Y. Kao, W. C. Carter, and Y. Chiang, Model for the particle size, overpotential, and strain dependence of phase transition pathways in storage electrodes: Application to nanoscale olivines, Chemistry of Materials, 21 (2009), pp. 1557-1571. 